Rev Biomed 2001; 12:35-45.

\title{
Salud-enfermedad reproductiva: una aproximación a los derechos reproductivos en Yucatán.
}

Revisión

Judith Ortega-Canto, Jolly Hoil-Santos, Andrés Santana-Carvajal, Guadalupe Andueza-Pech.

Laboratorio de Medicina Social, Centro de Investigaciones Regionales "Dr. Hideyo Noguchi", Universidad Autónoma de Yucatán. Mérida, Yucatán, México.

\section{RESUMEN.}

Objetivo. Caracterizar el perfil de saludenfermedad reproductiva para Yucatán.

Fuentes de obtención de la información. Instituto Nacional de Estadística, Geografía e Informática (INEGI) y Secretaría de Salud. Revistas de las Conferencias Internacionales (Cairo, 1994 y Beijin, 1995) sobre políticas de salud.

Resultados. El acceso a la salud y los derechos reproductivos está vinculado a la solución de rezagos sociales tales como desempleo $(47.2 \%)$, analfabetismo (15\%), entre otros. Las muertes femeninas durante el proceso reproductivo, seguidas de las muertes por cáncer cervicouterino y mamario, son prevenibles. Accidentes, tuberculosis y SIDA ocupan un lugar importante de este perfil, y afectan a uno u otro sexo. El aborto y la violencia de género son problemas que subyacen y que, junto a enfermedades congénitas, deficiencias en la calidad de atención médica, no se registran adecuadamente; menos se discuten y se atienden. Aspecto que recrudece para la población femenina, su condición marginal al interior de la marginalidad de otros.

Conclusiones. La importancia de nuevas política radica en su instrumentación local, partiendo de las diferencias socioculturales. Será importante fomentar cambios substanciales en la calidad de vida de los yucatecos; incorporar elementos innovadores en la búsqueda de nuevos indicadores; en las formas de registro estadístico; en la organización de servicios de salud; y, en las alianzas entre sector gubernamental y no gubernamental; mismas en las que la participación ciudadana adquiera un papel determinante en la transformación en materia de salud y derechos reproductivos.

(Rev Biomed 2001; 12:35-45)

Solicitud de sobretiros: Dra. Judith E. Ortega-Canto, Depto. de Medicina Social, C.I.R. "Dr. Hideyo Noguchi-", Universidad Autónoma de Yucatán, Ave. Itzáes No. 490 x 59, C.P.97000, Mérida, Yucatán, México. E-mail: ocanto@tunku.uady.mx. 


\section{J Ortega-Canto, J Hoil-Santos, A Santana-Carvajal, G Andueza-Pech.}

Palabras clave: salud reproductiva, epidemiología, derechos reproductivos, enfermedades de la mujer, Yucatán México.

\section{SUMMARY.}

Reproductive health-disease: a review about reproductive rights in Yucatan.

Objective. To characterize the reproductive healthdisease profile in Yucatan.

Source of information. National Institute of Statistics, Geography and Informatics (INEGI, Mex), National Health Services (Secretaría de Salud, Mex) and International Conferences (Cairo 1994 and Beiging 1995) about Health Policy.

Results. Access to health care and reproductive rights are related to social problems such as unemployment (47.2\%) and illiteracy (15\%) among others. The reproductive role of women also imposes particular stresses and risks on them for much of their lives. Death during pregnancy and childbirth as well as those caused by cervical and breast cancers are preventable. Abortion, violence and congenital diseases together with defficiencies in the quality of medical services are conditions which are not being seriously taken into account. Furthermore they are not discussed and in therefore cannot be solved. Health which is a fundamental right is still denied to many women specifically due to their marginal condition in Yucatan.

Conclusions. The importance of new polices is based on the fulfillment of local needs and takes into account the socio-cultural differences. It is important to encourage the improvement of women's well-being. The incorporation of new elements must be considered in order to look for new indicators of health, to develop better statistic records, organization of the health services and integration of govermental and non-governmental programs. In all those changes, all citizens will be able to play an active role in the transformation of their society especially on reproductive health and rights. (Rev Biomed 2001; 12:35-45)

Key words: Reproductive health, epidemiology, reproductive rights, women's diseases, Yucatan Mexico.

\section{INTRODUCCIÓN.}

Será importante partir del significado político y social del concepto de Salud Reproductiva, en la promulgación de derechos humanos. Más específicamente, de los derechos sexuales y reproductivos como el lente con el que habrá que leer los datos que en este artículo se analizan. "Estos derechos se basan en el reconocimiento del derecho básico de todas las parejas e individuos a decidir libre y responsablemente el número de hijos; el espaciamiento de los nacimientos e intervalo entre éstos; a disponer de la información y de los medios para ello y, el derecho a alcanzar el nivel más elevado posible de salud sexual y reproductiva" (1). El propósito del presente trabajo es iniciar un análisis respecto a si la población yucateca cuenta con los elementos necesarios para alcanzar niveles óptimos de salud reproductiva, y por ende, si se ejercen en Yucatán México, los derechos reproductivos.

\section{Algunos datos de Salud Reproductiva a nivel nacional.}

A nivel nacional en la República Mexicana, la mortalidad femenina es el talón de Aquiles de nuestro sistema de salud; no puede hablarse de un descenso notable durante la década de los noventa. De 5.5 muertes (por diez mil nacidos vivos registrados) en 1990, se redujo a 4.0 en 1994, para incrementarse de nuevo a 5.3 en 1996. Aunque aumentan las consultas prenatales y las usuarias de métodos anticonceptivos, la tasa de mortalidad materna se mantiene, de ahí que resulte poco acertado asentar que es la tasa de fecundidad, el factor asociado a las muertes maternas e infantiles. En cambio, es más probable que tengan relación directa con el número de abortos complicados, y con las complicaciones del embarazo, parto y puerperio. Éstas, sumadas a la mortalidad por cáncer cervicouterino ( 9.5 muertes por 100,000 mujeres en 1995), y mamario (6.6 muertes en

\section{Revista Biomédica}




\section{Salud-enfermedad reproductiva en Yucatán, México.}

1995), a la mortalidad por lesiones accidentales en población de 15 a 59 años, a la morbilidad por tuberculosis pulmonar y morbilidad por SIDA, son eventos que en conjunto conforman el perfil mórbido que impacta la vida de las mujeres.

Las tres últimas causas mencionadas, también abaten la vida de los hombres, grupo que tiene un papel de peso en el perfil de morbimortalidad femenina, dado el papel protagónico del varón como la contraparte de cada caso de embarazo, como facilitador o no de que la mujer acceda a algún tipo de control prenatal o a algún método de planificación familiar; como agente del bienestar familiar y/o como disruptor del mismo. Estos aspectos todavía no son tomados en cuenta por las fuentes oficiales.

Ahondando un poco más en el aborto, el Consejo Nacional de Población (CONAPO) informa que este problema ocupa la quinta y a veces la cuarta causa de las muertes femeninas. La Encuesta Nacional de la Dinámica Demográfica (ENADID, 1992) reporta que alrededor del $19.8 \%$ de las mujeres entre 15 y 49 años de edad, alguna vez embarazadas, habían experimentado un aborto. Según la Organización Panamericana de la Salud, en el Distrito Federal, la tasa de abortos asciende a 348 por 1000 mujeres embarazadas. Resultados que apuntan a que un $4.4 \%$ de las mujeres hospitalizadas en los servicios de salud, ingresaron por complicaciones de un aborto; que en un $8 \%$ los casos fueron reportados como inducidos. Asimismo se indica que existe un subregistro de 150 mil abortos inducidos, los cuales, en la medida que son efectuados de manera extrahospitalaria y/ o por manos no calificadas, se constituyen como riesgo de muerte; no es el acto quirúrgico en sí mismo, sino las circunstancias sépticas del mismo. Rainer Rosembaum director del Fondo de Población de las Naciones Unidas para México y Cuba (1995), declaró que "la despenalización del aborto es deseable en nuestro país, y que sería un importante segundo paso, ya que debe darse prioridad a la prevención de embarazos no deseados mediante una difusión adecuada y completa de los métodos anticonceptivos y la salud reproductiva" (2).

El aborto es actualmente un tema de discusión abierta a muchos niveles. Un resultado valioso de este proceso es la apertura de la institución médica para trabajar esta área problemática de la salud de las mujeres, silenciada por mucho tiempo por los programas de salud. El campo de la discusión ha logrado distanciarse de las posiciones conservadoras que pretenden dar respuestas morales a problemas sociales; de ahí la importancia de combatir la desinformación que promueven grupos llamados a veces "reaccionarios".

La violencia de género es otro aspecto que vive en el subregistro, pero que va ganando espacio en instituciones jurídicas, y se va convirtiendo en preocupación de grupos y organizaciones no gubernamentales. Por razones histórico-culturales y sociales, los crímenes que a diario se cometen contra niñas/os y mujeres, no son registrados como tales, fundamentalmente, porque las víctimas son mujeres. La vida de mujeres de todas las edades se ve amenazada de manera cotidiana por diversas formas de violencia y privación, que van desde la violación sexual hasta formas de abuso como la violencia doméstica, la prostitución forzada, la mutilación genital y los asesinatos de mujeres "por honor" (2).

Según la Organización de Naciones Unidas (ONU), la discriminación contra la mujer es descrita como: "toda distinción, exclusión o restricción, basada en el sexo, que tenga por objeto o por resultado menoscabar o anular el reconocimiento, goce o ejercicio por la mujer independiente de su estado civil, sobre la base de la igualdad del hombre y la mujer- de los derechos humanos y las libertades fundamentales en la esfera política, económica, social, cultural, civil, o en cualquier otra esfera" (3). De esta manera, la falta de acceso a información, servicios, derechos a elegir sobre el cuerpo, la pareja, la vida personal, familiar y social, debe ser reconocida como formas de discriminación, en los que se violan los derechos fundamentales de las mujeres. Este aspecto 
J Ortega-Canto, J Hoil-Santos, A Santana-Carvajal, G Andueza-Pech.

redunda directamente en los índices cuantitativos y cualitativos de la salud reproductiva y sexual femenina, así como en su desarrollo y desempeño como seres sociales.

\section{El panorama para Yucatán.}

Algunas características demográfícas y socioeconómicas.

Yucatán según el censo de 1995 tiene una población total de 1,556,662 habitantes, los cuales están distribuidos en tres grandes zonas: urbana (41.7\%), en otra zona urbana conformada por villas y pequeñas ciudades $(19.2 \%)$, y una rural (39\%). Los nacimientos han disminuido de 41, 495 en 1992, a 39, 226 en 1997 (4).

Del total de los habitantes entre 15 años y más, el 84.9\% (860,633 personas) son alfabetas; el $14.9 \%$ es analfabeta $(151,832)$, siendo mayor esta deficiencia para el grupo femenino que agrupa al 59.62\% de quienes no saben leer y escribir (4).

Un rezago notable es el desempleo, aunque las fuentes oficiales reporten que es una condición que sólo existe para un $2.9 \%$ de la población yucateca. Sin embargo, un análisis minucioso permite cuantificar que aproximadamente un $47.32 \%$ de la población total de la entidad sobrevive a formas claras o veladas de desempleo y subempleo. El 58.53\% de la población entre 12 años y más, es económicamente activa (PEA), en contraste a un $41.44 \%$ que es inactiva. Este último porcentaje ha disminuido, ya que para 1990 era de $55.34 \%$ (5). Por sexo, el $66.04 \%$ de la PEA pertenece al sexo masculino, y el $33.96 \%$ al sexo femenino. De manera predominante, es el sector terciario quien absorbe al $49.15 \%$ de la PEA; el primario al $26.23 \%$, y el secundario al $24.52 \%$ (5). Aunque de la PEA, el $94.7 \%$ se reporta como empleada, en el listado de tipos de ocupación se combinan categoría tales como: la de "trabajador por su cuenta" (33.48\%), y la de "trabajador familiar sin pago" (10.94\%) (5), que sumados señalan que un $44.42 \%$ de la población desarrolla estrategias de subsistencia económica, situación que ilustra la condición de probable subempleo, y

\section{Cuadro 1}

Distribución porcentual de empleo, subempleo y desempleo en estado de Yucatán, 1995

\begin{tabular}{lc}
\hline & Porcentaje \\
\hline Personas con empleo & 52.7 \\
Subempleo: & 33.5 \\
"Trabaja por su cuenta" & 10.9 \\
"Trabaja con familiar sin pago" & 2.9 \\
Desempleo & 100.0 \\
\hline Total & \\
\hline
\end{tabular}

Fuente: INEGI, Perfil Sociodemográfico, 1995.

la limitación del sector oficial para absorber mano de obra (cuadro 1).

Detallando aún más, mujeres y jóvenes son los dos grupos más afectados. Las tasas de desempleo en población de 12 años y más, siempre son más altas para el sexo femenino; así tenemos que mientras el $21.2 \%$ sucede en los varones, es el $61 \%$ en las mujeres quienes lo enfrentan. Seguidos del grupo de jóvenes entre 12 y 24 años de edad. La población económicamente inactiva se distribuye en dos grupos fundamentales: quienes se dedican a los quehaceres del hogar, y los estudiantes (32.6\%). Las causas del desempleo son: el cese del trabajador, la insatisfacción con el trabajo presentado o entregado; o porque de antemano, son trabajos temporales (4).

Resulta llamativo que coexista la desocupación con nivel de escolaridad media en el área urbana. Predomina la secundaria incompleta o completa $(47.4 \%)$, seguida del grupo que cursó la primaria completa $(23.1 \%)$, y luego el grupo que ha cursado la enseñanza media superior y superior (21.5\%). Según la posición en el hogar, estas personas son "hijos" (56.4\%) y "jefes del hogar" (26.2\%), seguido del grupo de "cónyuge" (11.1\%) (4).

El $34.86 \%$ de la población sobrevive con menos de un salario mínimo (5). Si a la población de desocupados o semi-desocupados que se había mencionado (47.22\%), se agrega la población ocupada que recibe un ingreso insuficiente, es

\section{Revista Biomédica}




\section{Salud-enfermedad reproductiva en Yucatán, México.}

posible imaginar que más de las tres cuartas partes de la población yucateca pueda estar viviendo en condiciones de marginalidad social.

Como indicador positivo aparece el crecimiento de la tasa de viviendas y los servicios con los que cuentan. De 1970 a 1990 fue de 3.8\%; entre 1990 y 1995 muestra un ligero descenso $(3.3 \%)$; porcentaje que continúa siendo mayor a la tasa de crecimiento poblacional del 2.4 por ciento. Respecto a los servicios con los que cuentan las viviendas: el $94.29 \%$ cuentan con luz eléctrica; el $54.81 \%$ con servicio sanitario; drenaje en un $53.73 \%$; y agua entubada en un $85.82 \%$ (5).

La jefatura de los hogares también se ha transformado para aparecer como un factor de riesgo económico. De los 338, 581 hogares que para 1995 tenía el Estado, el 85\% eran de jefatura masculina, y el $15 \%$ femenina (5), circunstancia difícil para estas familias, dada la posición de marginalidad con que las mujeres aún son vistas en la gran mayoría de los ambientes laborales.

Respecto a seguridad social, sólo el $39.13 \%$ de la población yucateca es derechoahabiente de algún servicio de salud; el $60.84 \%$ no cuenta con esta reivindicación social (4). Aunque en el discurso y organización de los servicios de salud del Instituto Mexicano del Seguro Social, se dice haber ampliado la cobertura a través de la modalidad de "solidariohabientes", habría que constatar si ello se ha traducido en mayor infraestructura y recursos médicos y de enfermería, o sólo se han abierto las puertas para quienes lo soliciten. Si es así, la carga de trabajo para el personal de salud traducido en demanda cada vez mayor de pacientes a los que hay que atender con los mismos recursos físicos y humanos, deben estar generando una consecuente disminución de la calidad de atención. Aunque se le atribuye a la Secretaría de Salud, la cobertura del 66.1\% (1994) (7) de los usuarios de los servicios de salud, es sabido que sus instituciones no cuentan con los recursos necesarios para ofertar atención de calidad. De ahí, que la información oficial esté disfrazando otro rezago importante que debería ser un derecho de cada ciudadano.

\section{Salud-enfermedad-atención reproductiva en Yucatán.}

En este apartado se describen y analizan los indicadores que informan a nivel oficial, de los patrones reproductivos de la población, frecuencia y/o índice de la morbilidad y mortalidad en la etapa reproductiva.

Fecundidad.- Como reflejo de lo que sucede con la dinámica poblacional a nivel nacional, Yucatán tiene una pirámide poblacional que crece a expensas de los niños y los adolescentes, para luego adelgazarse a partir de los grupos de 25 a 29 años y mayores. Haciendo un análisis de la fecundidad en la entidad, se estima según la ENADID, un total de 702 mil mujeres, de las cuales 339 mil están en edad fértil, es decir, que tienen entre 15 y 49 años de edad (cuadro 2). Una de cada tres mujeres tienen entre 20 y 29 años de edad, periodo dentro del cual se alcanza un mayor nivel de fecundidad. Sobre el estado civil de las mujeres en edad fértil, el $61.7 \%$ están casadas o unidas, el $33.7 \%$ son solteras y el $4.6 \%$ son separadas, divorciadas o viudas (6).

Se observa que en el intervalo de edad entre 15 a 24 años queda concentrado el $42.14 \%$ de la población femenina en edad fértil. Para 1992, el promedio de hijos nacidos vivos era de 2.3 hijos

\section{Cuadro 2}

Distribución porcentual de la población femenina en edad fértil por grupos quinquenales de edad, 1992

\begin{tabular}{crc}
\hline Grupos de edad & Nacional & Yucatán \\
\hline $15-19$ & 22.8 & 24.7 \\
$20-24$ & 18.7 & 18.7 \\
$25-29$ & 15.7 & 14.2 \\
$30-34$ & 13.8 & 11.4 \\
$35-39$ & 12.1 & 12.9 \\
$40-44$ & 9.1 & 10.8 \\
$45-49$ & 7.8 & 7.3 \\
Total & 100.0 & 100.0 \\
\hline
\end{tabular}

Fuente: INEGI, Encuesta Nacional de la Dinámica Demográfica, 1992.

Vol. 12/No. 1/Enero-Marzo, 2001 


\section{J Ortega-Canto, J Hoil-Santos, A Santana-Carvajal, G Andueza-Pech.}

por mujer, mismo que coincide con el valor a nivel nacional. Este promedio se incrementa conforme avanza la edad de las mujeres: mientras las mujeres entre 20 y 24 años han tenido poco menos de un hijo nacido vivo, las que están por terminar su periodo reproductivo que tienen entre 45 y 49 años, han tenido poco más de cinco hijos. Por lugar de residencia existe cierta diferencia entre mujeres rurales y urbanas: 2.7 hijos nacidos vivos para las primeras, y 2.2 para las segundas (6). Aspectos que informan de una mayor paridad en las mujeres urbanas yucatecas comparadas con la paridad de mujeres urbanas a nivel nacional. Asimismo, una menor paridad de las mujeres rurales yucatecas comparadas con las mujeres rurales a nivel nacional. Condición que informa una mayor eficiencia del programa de planificación familiar, en el área rural yucateca.

Yucatán ocupa el decimotercer lugar por tasa de fecundidad en el país, después de los estados de Chiapas, Oaxaca, Guerrero, Puebla, Zacatecas, Michoacán, Querétaro, San Luis Potosí, Durango, Guanajuato, Tlaxcala y Jalisco. Las mujeres yucatecas muestran una descendencia de 3.8 hijos por mujer, valor que supera al promedio nacional (3.5 hijos) (6).

Anticoncepción.- La anticoncepción como la práctica que dentro del programa de planificación familiar ha sido instituida como determinante en el descenso de la fecundidad, fue motivo de estudio a través de la ENADID. Se exploró el conocimiento y uso de métodos anticonceptivos en mujeres entre quince a cuarenta y nueve años. Los resultados mostraron que aunque se ha incrementado el número de usuarias de métodos anticonceptivos modernos, se mantiene una alta proporción de usuarias de métodos tradicionales y poco efectivos como son: el ritmo y el retiro. El patrón de conocimiento de medios para regular la fecundidad en el estado de Yucatán es similar al del país, aunque en una escala menor. Los métodos más conocidos son las pastillas seguidos de la operación femenina, las inyecciones y el dispositivo uterino (DIU). El preservativo es conocido por el
36.7\% de la población femenina. Los más usados resultaron ser: las pastillas, la operación femenina y el DIU. Es notable la baja presencia de la operación masculina (6).

A mayor nivel de uso de métodos anticonceptivos modernos, más bajo nivel de fecundidad. Yucatán es uno de los estados de bajo uso de éstos métodos, lo cual se ve reflejado en su tasa global de fecundidad (3.8 hijos por mujer); asimismo, es la sexta entidad de la República que utiliza en gran medida, métodos tradicionales, sólo superado por Oaxaca, Puebla, Guanajuato, Michoacán y Chiapas (6). En el grupo de mujeres actualmente usuarias, a la mayor parte se les ha practicado la operación femenina (40.8\%); les siguen las que utilizan pastillas con el $27.5 \%$, y las que emplean métodos tradicionales como el ritmo o el retiro, con el 19.2\%. La mayor proporción de uso de métodos anticonceptivos se concentró en mujeres que tenían tres hijos actualmente vivos; esto parece informar que tres hijos es la estructura numérica ideal para la generación actual.

Respalda lo anterior la cobertura menor del uso de métodos anticonceptivos en el Estado (36.7\%) en contraste a lo reportado a nivel nacional (40.2\%). Cuando el análisis se centra en las mujeres que se encuentran en algún tipo de unión marital, se tiene que el $56.2 \%$ están planificando su familia, mientras que a nivel nacional lo hace el $63.1 \%$ (6). Las mujeres yucatecas en promedio tienen tres hijos (1990) (7), lo cual comparado con la media nacional de 2,37 (1996) (8) evidencia el rezago respecto a estas prácticas. Sin embargo, la eficiencia de los programas institucionales es evidente para el área rural en Yucatán, al mostrar que para las mujeres de estas zonas se registra una menor fecundidad respecto a sus pares de las zonas rurales a nivel nacional (figura 1).

Mortalidad general, infantil y materna.El análisis de la mortalidad lleva implícita una dificultad al interior de nuestro sistema de registro estadístico, dada la variedad de fuentes y formas de clasificar y ordenar las causas de defunción por cada institución. Como denominador común, es

\section{Revista Biomédica}


Salud-enfermedad reproductiva en Yucatán, México.

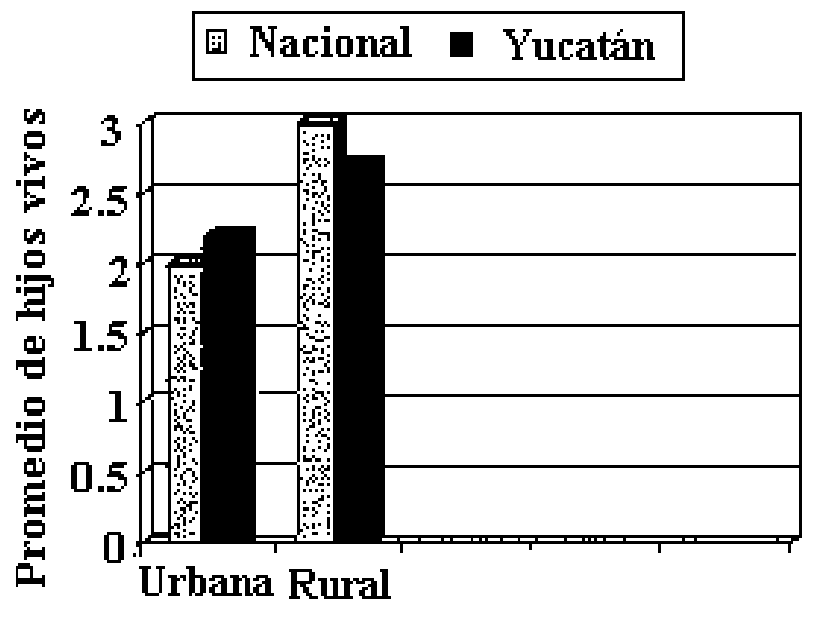

Fuedte: REGLI Euneta Hacional dela Tíámica Denoprifica, 1992

Figura 1.- Promedio de hijos nacidos vivos de la población femenina en edad fértil según área de residencia, 1992.

posible reconocer que se transforma a través del tiempo para hacernos parecer una sociedad en transición. Por ejemplo, la tasa de mortalidad materna ha disminuido de 9.5 (por mil nacidos vivos) en 1980 a una de 5.3 para 1995. La tasa de mortalidad general es de $5.2 \%$ (por mil habitantes); la infantil es de 18.4 (por mil nacidos vivos). La esperanza de vida para ese mismo año es de 72.3 años promedio (9).

Cuando se revisa con minucia el listado completo de las causas de muerte en la entidad, aparecen las enfermedades degenerativas y la violencia combinadas con las enfermedades infecciosas y parasitarias, los accidentes obstétricos y perinatales, como lo llamativo en la lista de las veinte principales causas de muerte en Yucatán. En la mortalidad hospitalaria reportada por el IMSS, aparecen la diabetes mellitus y la cirrosos hepática, como causas predominantes y más frecuentes que el infarto y demás degenerativas. Lo llamativo es que un $55.1 \%$ de causas fueron agrupadas como "Resto de causas". Aún informes de la ENADID (1992) cuando lista la mortalidad a nivel nacional, aparece un $60 \%$ de causas incluidas en el rubro de "Las demás". Esta lectura desdibuja del panorama de la muerte, una serie de causas que se hacen ver como poco frecuentes o dañinas. Sin embargo, haciendo una revisión y una suma de las mismas, pudo constatarse que son un conjunto de causas que abaten principalmente a la población infantil y materna, y que muy probablemente, serían más frecuentes que las causas que aparecen como predominantes en nuestras estadísticas oficiales.

Aunque la mortalidad infantil ha disminuido de manera notable, es necesario reconocer como un problema pendiente, las causas que pueden ser prevenibles. Son los niños varones menores de una semana (28\%) y de 1 a 11 meses (22\%), los que ocupan los dos primeros lugares de muerte infantil; seguidos de las niñas de ambos grupos de edad. Entre las principales causas de mortalidad en menores de un año (1995) fueron reportadas: "ciertas afecciones originadas en el periodo perinatal", seguidas cercanamente por las "anomalías congénitas"; en tercer lugar, la "neumonía e influenza" seguida de las "enfermedades infecciosas intestinales" (9).

$\mathrm{El}$ análisis minucioso permite saber a qué tipo de problemas hace referencia la causa llamada: "ciertas afecciones en el periodo neonatal", la cual, no sólo incorpora una amplia variedad de afecciones, sino por la frecuencia de cada una de ellas, son un llamado de atención a nivel del desarrollo de nuestro país, en la medida que la muerte infantil continúa siendo un azote. Tenemos diagnósticos tales como: anomalías congénitas; espina bífida e hidrocefalia; fisura del paladar y labio leporino; otras deformidades del aparato digestivo; resto de anomalías congénitas; perinatal; enfermedades de la madre que afectan al feto o al recién nacido; complicaciones obstétricas que afectan al feto o al recién nacido; crecimiento fetal lento; desnutrición e inmadurez fetal; traumatismo del nacimiento; hipoxia, asfixia y otras afecciones respiratorias del feto o del recién nacido; enfermedad hemolítica del feto o del recién nacido; resto o ciertas afecciones originadas en el periodo perinatal; síndrome de la muerte súbita del lactante; falla respiratoria. En conjunto, problemas que atañen a deficiencias en aspectos como: el consejo 


\section{J Ortega-Canto, J Hoil-Santos, A Santana-Carvajal, G Andueza-Pech.}

genético, las condiciones nutricionales, ambientales y de salud en general de las mujeres en edad reproductiva, a la calidad de los servicios de atención obstétrica, y a la cultura en salud de las mujeres y sus parejas masculinas. Problemática vinculada directamente a la pobreza y al desconocimiento de los derechos reproductivos.

Ahora bien, sucede lo mismo cuando la revisión agrupa al conjunto de causas para los grupos en edad reproductiva, fundamentalmente, el grupo femenino. Problemas infecciosos, crónicoinflamatorios, prácticas sépticas, complicaciones y calidad deficiente en la atención en servicios de salud, parecen explicar el siguiente listado: enfermedades de los órganos genitales masculinos; enfermedades de los órganos genitales femeninos; salpingitis y ooforitis; enfermedades inflamatorias del tejido celular pelviano y del peritoneo; enfermedades inflamatorias del útero, de la vagina y de la vulva; prolapso utero-vaginal; esterilidad de la mujer; resto de enfermedades de los órganos genitales femeninos; aborto; aborto inducido legalmente; resto de aborto; causas obstétricas directas; hemorragia del embarazo y del parto; toxemia del embarazo; infecciones del aparato genitourinario en el embarazo; parto obstruído; complicaciones del puerperio; resto de causas obstétricas directas; causas obstétricas indirectas; y hasta el parto normal aparece como causa de muerte.

La amplia gama de causas de muerte impide un análisis desde el mismo sistema estadístico, enmascara la frecuencia con que en realidad se mueren las mujeres y los niños, así como la naturaleza de sus causas. La tipificación en sí misma resulta muchas veces imprecisa, y llama la atención, que no se reporten los casos de muerte por cáncer. ¿Será que no se reportan porque las muertes suceden con cierta frecuencia en los domicilios, y cuando suceden, los servicios de salud no los registran?

A manera de conclusión, es importante reconocer que existe un riesgo reproductivo de gran magnitud durante el proceso reproductivo femenino, mismo que atañe al proceso de crecimiento fetal, su nacimiento y su vida perinatal y neonatal. Conveniente sería para nuestro sistema de registro estadístico, incorporar en su tipificación, un rubro que incorporara "causas de muerte durante el proceso reproductivo" y distribuírlas por grupos de edad y sexo, en la medida que pueda reconocerse que tanto las mujeres como los hombres informan de la salud reproductiva. Asimismo, para englobar los riesgos y complicaciones antes, durante y después del embarazo; parto y puerperio; complicaciones del aborto y la anticoncepción; las cuales agrupadas, muy probablemente conformarían una frecuencia igual, semejante y hasta mayor a las causas que aparecen como predominantes en el perfil de morbi-mortalidad para Yucatán.

En general, la falta de especificación impide un análisis más riguroso de las causas por las que la gente se enferma y muere. Ello por supuesto, redunda en un sesgo importante para el análisis de nuestros perfiles de salud/enfermedad, así como, cuando se quiere pensar en programas de control, de prevención y hasta de erradicación.

Mortalidad por cáncer cervicouterino.Lo disperso de la información para las enfermedades neoplásicas como causa de muerte impidió obtener un perfil para fines de este artículo, por lo que nos limitamos a seleccionar algunos de los resultados de un estudio (10), a manera de contar con una primera aproximación del comportamiento del cáncer cervicouterino para Yucatán.

El comportamiento de la morbilidad por neoplasias en Yucatán muestra claramente que después de las de piel, siguen las de cérvix y mama con las tasas más altas. De una revisión hecha de 771 expedientes clínicos del Centro Anticanceroso de Mérida (10), de mujeres que acudieron a consulta por primera vez en el periodo de enero de 1990 a diciembre de 1995, fue posible encontrar un promedio estable de 125 casos anuales de cáncer cervicouterino, que informan que dicha enfermedad no ha disminuido desde 1990. La rango de edad 


\section{Salud-enfermedad reproductiva en Yucatán, México.}

en la que predominó la enfermedad fue entre los 45 y 54 años de edad (23.9\%).

Cuando la autora exploró los antecedentes personales heredofamiliares, no encontró asociación con consumo de tabaco, ni fuerte antecedente de cáncer, más que en un $2.18 \%$ (13 casos). En 131 expedientes en los que se consignó la búsqueda de diabetes mellitus tipo II, se reportó como positiva en el $43.5 \%$ de este grupo; once padecían hipertensión arterial $(8.36 \%)$, y sólo cuatro pacientes tuvieron antecedentes de algún otro cáncer primario $(3 \%)$.

Un predominio de inicio de relaciones sexuales coitales antes de los veinte años, fue seguida de que: en 84 expedientes (14\%) en los que se había registrado el número de compañeros sexuales, se encontró que más de la mitad (67.8\%) reportó uno solo, mientras que en el $32.1 \%$ restante, dos o más. La multiparidad (cuatro partos y más) aparece como un factor asociado de manera importante en el $84 \%$ de estas pacientes. De esta manera, la alta paridad aparece como un factor que puede estar predisponiendo.

Solís V (10) informó que en los expedientes en los que sí estaba consignada la ocupación (561 pacientes) y el estado civil (759 pacientes), en su gran mayoría fueron mujeres dedicadas a las labores del hogar, casadas. En mucha menor proporción estaban las viudas (92 casos $=15.46 \%$ ), las solteras (26 casos $=4.37 \%)$, y en unión libre (21 casos $=3.53 \%)$. Dada la población socioeconómica a la que atiende el centro Anticanceroso, la gran mayoría (90.12\%) correspondió a nivel socioeconómico bajo (493 casos) y su proveniencia fue rural en un $60.1 \%$ (349 pacientes), y urbana en un $39.9 \%$ (231 pacientes). Con pertenencia al estado de Yucatán en un $60.3 \%$ (350 casos), y con residencia en la ciudad de Mérida un 26\% (91 pacientes). Vale la pena mencionar también, que este centro recibe pacientes de unidades de los servicios institucionales de salud en un $70 \%$ de los casos, y del sector privado en un $30 \%$. Pobreza, ruralidad, falta de acceso a servicios, ejercicio coital temprano, y alta paridad fueron las características sobresalientes en las mujeres de este estudio.

\section{Consideraciones finales.}

Las fuentes oficiales de información constituyen para todo estudioso de la realidad social, un buen punto de partida. De ahí que la búsqueda de la calidad de información que ofertan ha sido en la última década, una preocupación substancial para dichas instituciones. Sin embargo, es importante reconocer, que los indicadores actuales relativos a salud reproductiva, miden casi exclusivamente, los daños a la salud. Daños concretados en enfermedad y muerte cuya distribución muestra a una sociedad homogénea, por la forma como los datos son presentados. Aspectos tales como las diferencias rural-urbano no son consideradas. La pertenencia socioeconómica tampoco aparece relevante para hablar de la distribución de la enfermedad y la muerte. Menos, las diferencias socio-culturales y de género.

Por otro lado, los datos presentados validan la importancia de no dejar de lado, las deficiencias en las que transcurre la vida de muchas mujeres y hombres. Sobresalen: condiciones de subempleo o desempleo y una PEI (población económicamente inactiva) a pesar de tener niveles medios de escolaridad; la precariedad de las viviendas de ciertos grupos de la población; la inaccesibilidad a los servicios de atención en porcentajes importantes; hogares en los que se incrementa la jefatura femenina; la violencia en general y de género en particular. En conjunto, son características del contexto yucateco que entorpecen los programas de mejoramiento a la salud pública, mientras que dichas condiciones no son transformadas a favor de una población que tenga cubierto un mínimo de bienestar material, como la estrategia que favorecería una conciencia en salud reproductiva.

El negar que la realidad social es vivida de diferentes maneras, de acuerdo al conjunto social al que se pertenece, hace que la importante gama de recursos físicos y humanos no reditúe en el abatimiento de problemas específicos de grupos que deberían ser prioritarios; y que ocurran eventos 


\section{J Ortega-Canto, J Hoil-Santos, A Santana-Carvajal, G Andueza-Pech.}

penosos como la muerte de mujeres y niños por enfermedades prevenibles.

Nuestro fin de milenio deja sentir -a través de las conferencias internacionales de población-, una conciencia mundial del riesgo reproductivo asociado a la pobreza, a la inaccesibilidad a la ciencia y a la tecnología, y a la inequidad de género. Los derechos sexuales y reproductivos como punta de lanza, proponen la dimensión moral que ha estado ausente en nuestros programas y políticas poblacionales. Si queremos una "maternidad sin riesgos", tendremos que unificar criterios para implementar programas de nutrición, vivienda, escolaridad, educación para la salud, acceso a empleos remunerados, la vigilancia de los ecosistemas familiares y laborales; acceso a servicios de salud; todos y cada uno de ellos, factores que exponen a las mujeres a riesgos durante el embarazo y el parto.

El registro de las muertes maternas deben poder informar del lugar marginal y de la probable condición de explotación que vivió cada fallecida. Si es cierto que el embarazo y el parto es importante atenderlos, de igual importancia será el entorno en el que se dan dichas etapas, y la responsabilidad compartida con los esposos, hijos e hijas mayores; la vigilancia estrecha de enfermeras y trabajadoras sociales; la participación de las parteras, entre otras.

El aborto no reportado, es un evento que toca las fibras morales de nuestra sociedad; y la postura indiferente de nuestro sector salud, facilita u obliga a la clandestinidad. Será importante reconocer que es producto de los embarazos no deseados a los que las mujeres están constantemente expuestas por razones tales como: falta de información, violencia sexual, falta de acceso a servicios de salud, falta de colaboración de la pareja, ineficacia del método empleado, uso inadecuado de medidas anticonceptivas, entre otras. Por una u otra razón, las mujeres no son capaces de planificar su familia como ellas mismas quisieran.

El aborto es un evento complicado; pero, la mayoría de las veces, lleva implícita la desesperación femenina como característica determinante de esta práctica. Sino, ¿cómo entender que las mujeres decidan someterse a procedimientos quirúrgicos en manos no calificadas, a sabiendas que pueden perder la vida; $\mathrm{o}, \tan$ dolorosos y violentos como inyectarse o tomar abortivos, introducirse sondas, palos, ramas, tallos, agujas, substancias cáusticas, sufrir caídas o golpes, como las más conocidas? (11).

La salud reproductiva vista como un proceso, y no como apartados definidos desde la perspectiva médica-institucional, reorientaría los gastos en salud; la prevención de daños por ejemplo, haría posible a futuro, la medición de indicadores positivos. Si es cierto que "el 90 porciento de las muertes maternas son evitables, su persistencia es éticamente inaceptable" (11). Esta misma visión dinámica implicaría la definición de políticas a favor de una atención temprana: a la joven y al joven desde el inicio de la pubertad, a partir del momento que dejan de ser atendidos por los servicios de pediatría. Acciones y programas sobre adolescencia, y su salud sexual y reproductiva serían la puerta de entrada para la salud reproductiva de la mujer adulta y su pareja.

A la práctica médica se le ha delegado la responsabilidad, y/o ella ha monopolizado todo lo referente a salud/enfermedad/atención. ¿Esto tiene carácter ideológico? ¿Dónde se deja la capacidad de la ciudadanía, y la de instituciones gubernamentales y no gubernamentales? Nuestro sector salud aparece como responsable de las muertes por cáncer, por abortos complicados, por hemorragias y toxemias, entre otras; como si la población no tuviera ingerencia o derechos. ¿Cómo lograr la ansiada maternidad sin riesgos, si no partimos de que esta noción tendría que ser una consigna colectiva?. La sociedad debería conocer de las muertes de tantas mujeres para generar una conciencia que, -dolorosa inicialmente-, nos llevaría a retroalimentar nuestra propia cultura en salud, con el postulado de que la vida y la salud de las mujeres, tiene que empezar

\section{Revista Biomédica}




\section{Salud-enfermedad reproductiva en Yucatán, México.}

por ser algo valioso para el propio género femenino, e iniciar un proceso de transformación en la desigual interacción hombre-mujer (12).

$\mathrm{El}$ análisis de la salud reproductiva tendrá que incorporar las voces de las mujeres y los hombres, para conocer las particularidades ilustradas por las semejanzas, diferencias y hasta antagonismos entre los grupos humanos de un conjunto social a otro, así como el componente vivencial del ser hombre o mujer (13). De esta manera, los datos estadísticos que caracterizan el contexto donde sucede la saludenfermedad-atención reproductiva, se verían enriquecidos con la manera como los grupos humanos perciben, entienden y atienden el proceso reproductivo (estudios cualitativos), mismo que constituye en nuestra cultura, una etapa común y repetida de nuestras vidas.

\section{AGRADECIMIENTOS.}

Convenio de apoyo con la Fundación Ford, clave CIRB97-005.

\section{REFERENCIAS.}

1.- Sidney GN. Prefacio en: Acción para el Siglo XXI. Salud y Derechos Reproductivos Para Todos. Nueva York.: Family Care International; 1994. p. i-iv.

2.- GIRE, No. 6. Boletín trimestral sobre reproducción elegida. México, D.F. 1995. p. 3-8.

3.- Human Rights, the new concensus. London: Regency Press. 1994; p. 77-9.

4.- INEGI. Yucatán. Resultados definitivos; Tabulados Básicos; Tomo I. Conteo de Población y Vivienda. México: 1995. p. 99, 102, 136, 137, 144, 145, 164, 165, 226.

5.- INEGI. Perfil Sociodemográfico. México; 1995. p. 35, 40, 42, 44, 51, 57, 65. México.

6.- INEGI. Encuesta Nacional de la Dinámica Demográfica. México; 1992.

7.- INEGI. Niveles de bienestar. México; 1990. p. 198200.

8.- OPS La salud de las Américas. Washington D.C.
Publicación Científica 569. Volumen II. 1998. p. 404.

9.- Información Básica del Estado de Yucatán. Sistema Nacional de Salud. 1994. http://cenids.ssa.gob.mx/mapas/ yucatán.html.

10.- Solis V. Estudio Epidemiológico de Cáncer Cervicouterino en el Centro Anticanceroso (1990-1995). Tesis de licenciatura en Medicina de la Facultad de Medicina de la Universidad Autónoma de Yucatán. Mérida Yucatán, México. 1998; p. 33-36.

11.- Aborto clandestino: una realidad latinoamericana. 1994. p. 5-6. New York and Washington: The Alan Gutmacher Institute.

12.- The Alan Gutmacher Institute. La Práctica del Aborto Clandestino. 1994. P: 14-5. New York and Washington.

13.- Fundación Ford. Ortega-Canto J. Programa de Apoyo al Desarrollo Académico de Investigadores de la Salud Sexual y Reproductiva de la Universidad Autónoma de Yucatán”. Informe técnico. 1997. Mérida Yucatán, México. Clave CIRB - 97- 005. 